\title{
Simplified Sizing of Low-Density Lipoprotein Using Polyacrylamide Gradient Gel Electrophoresis of Plasma
}

\author{
Justin Westhuyzen ${ }^{1}$, Scott D. Graham ${ }^{1}$, Rohan L. Rasiah ${ }^{1}$ and David Saltissi ${ }^{2}$ \\ 1 Conjoint Internal Medicine Laboratory, Royal Brisbane Hospital, Herston, Brisbane, Australia \\ 2 Department of Renal Medicine, Royal Brisbane Hospital, Herston, Brisbane, Australia
}

\begin{abstract}
Summary: Low-density lipoprotein (LDL) particles can be separated into subfractions according to size by nondenaturing polyacrylamide gradient gel electrophoresis. Established research methods require specialised equipment and are frequently unsuited to the clinical laboratory. In this study, we utilised a colour flat bed scanner in conjunction with shareware image analysis software to compare LDL particle diameters of isolated LDL with LDL in whole plasma. LDL was isolated by ultracentrifugation and electrophoresed on 3-13\% gels (Gradipore; Sydney, Australia) for 2400 Volt-hours in parallel with plasma and molecular size standards. Coomassie Blue-stained gels were scanned in reflexive mode using a colour flat-bed scanner and Adobe Photoshop 3.0 software. Density traces of each lane were obtained using NIH Image software (public domain, USA). LDL particle diameters were determined from calibration curves of the log of molecular diameter of standards against migration distance. There was a good correlation between LDL particle diameters obtained using isolated LDL and whole plasma $(r=0.87$, $\mathrm{P}<0.001 ; \mathrm{n}=22)$. However, the group means $( \pm$ S. D. $)(24.7 \pm 0.6$ and $24.8 \pm 0.5 \mathrm{~nm}$ respectively) were statistically different on the paired $t$-test $(\mathrm{P}<0.05)$. It is unclear whether this numerically small difference is due to alterations in LDL during the longer preparative procedures for LDL, or to matrix effects during electrophoresis of plasma samples. In conclusion, plasma samples stained with Coomassie Blue and scanned with a colour flat bed scanner can conveniently be used for LDL particle sizing by non-denaturing polyacrylamide gradient gel electrophoresis.
\end{abstract}

\section{Introduction}

Elevated and/or abnormally modified serum low-density lipoprotein (LDL) cholesterol is a key risk factor in the development of atherosclerosis. Currently, attention has focussed on LDL particle subclasses, since population studies suggest that a predominance of small, dense LDL is associated with an increased risk of coronary artery disease $(1-5)$. LDL particle subclasses may also be important in the secondary dyslipidaemia of disease states such as chronic renal failure (6).

LDL particles can be separated into subfractions according to flotation characteristics using equilibrium density ultracentrifugation (7). However, the development of non-denaturing polyacrylamide gradient gel electrophoresis has greatly facilitated the investigation of LDL subpopulations $(8,9)$. In this technique, whole plasma or isolated LDL samples are electrophoresed on a gradient polyacrylamide gel, which in LDL studies is generally $2-16 \%$. Charged particles migrate through the cross-linked matrix until they reach their effective size exclusion limit, which is determined by their size and shape. The gels are then stained for either lipids or proteins, and scanned to obtain a densitometric tracing of the sample lane. Standards of known diameter are included on every gel for calibration purposes.
Densitometry of stained gels is generally undertaken using laboratory equipment such as dedicated gel densitometers and gel scanning attachments to spectrophotometers. To accommodate the different staining characteristics of protein and lipid, specific wavelengths are used: $596 \mathrm{~nm}$ for proteins stained by Coomassie G-250, $555 \mathrm{~nm}$ for Coomassie R-250, and using the lipid stain Oil Red O, $530 \mathrm{~nm}$ for lipids and $280 \mathrm{~nm}$ for proteins and latex particles (9). Colour flat bed scanners, although lacking specific wavelength selection, may provide a convenient and cheaper alternative for scanning gels. In the final step, a densitometric trace of each sample lane is obtained using commercially available software.

To facilitate the sizing of LDL particles, we developed simplified methods for use in the clinical laboratory. We utilised a colour flat bed scanner in conjunction with freely available image analysis software on a Macintosh computer to scan Coomassie blue-stained gels. Since the isolation of LDL by ultracentrifugation involves expensive centrifuges and lengthy procedures, we also compared LDL particle diameters obtained using isolated LDL with those obtained using whole plasma.

\section{Materials and Methods}

Subjects

Test subjects consisted of apparently healthy hospital workers, active elderly women and patients with end-stage renal failure who 
are at risk of accelerated atherosclerosis $(n=22)$. Informed consent was obtained from all subjects, and the study was approved by the Human Ethics Committee of the Royal Brisbane Hospital.

\section{Methods}

Blood was drawn by venipuncture after an overnight fast into evacuated tubes containing disodium EDTA $(1 \mathrm{~g} / \mathrm{l})$. Plasma was separated by low speed centrifugation and stored at $4{ }^{\circ} \mathrm{C}$ for $<2 \mathrm{~d}$ before electrophoresis. LDL was isolated without delay by a rapid ultracentrifugation method (10). In brief, plasma density was adjusted to $1.24 \mathrm{~kg} / 1$ with $381.6 \mathrm{~g} / 1$ solid $\mathrm{KBr}$ and layered under 2 volumes phosphate buffered saline (density $1.006 \mathrm{~kg} / \mathrm{l}$ ) in $5 \mathrm{ml}$ polyallomer centrifuge tubes. After centrifugation at 100000 (frequency, $\left.\min ^{-1}\right)(543,000 \mathrm{~g})$ for $120 \mathrm{~min}$ at $12^{\circ} \mathrm{C}$ in a Beckman TL100 ultracentrifuge (TLA100.4 rotor; Beckman, Palo Alto, CA, USA), the LDL band was aspirated through the wall of the centrifuge tube. Isolated LDL was stored at $4{ }^{\circ} \mathrm{C}$ in microfuge tubes for $<2 \mathrm{~d}$ before electrophoresis.

\section{Electrophoresis}

Electrophoresis of isolated LDL and plasma was carried out at $10^{\circ} \mathrm{C}$ using non-denaturing polyacrylamide gradient gel electrophoresis on $3-13 \%$ minigels $(72 \times 72 \times 3 \mathrm{~mm}$ ) (Gradipore Ltd, Riverside Corporate Park, 35-105 Delhi Road, North Ryde, Sydney 2113, Australia) (9). Samples were mixed with 2.5 volumes " $2 \mathrm{X}$ sample buffer" containing tracking dye (Coomassie blue) and sucrose (Gradipore Ltd) and 1.5 volumes electrophoresis buffer, $\mathrm{pH}$ 8.4. Aliquots $(10-15 \mu \mathrm{l})$ were applied to alternate lanes. Gels were electrophoresed in Tris-boric acid-EDTA buffer, $\mathrm{pH} 8.3$ (8) at $70 \mathrm{~V}$ for 15 min and then $150 \mathrm{~V}$ for a total of $16 \mathrm{~h}(2400 \mathrm{~V}$-hours) using equipment supplied by Gradipore Ltd. Gels were fixed in sulphosalicylic $\operatorname{acid}(0.1 \mathrm{~kg} / \mathrm{l})$ for $30 \mathrm{~min}$, and stained in Coomassie Blue G-250 for $4 \mathrm{~h}$ (Gradipure; Gradipore Ltd). Gels were destained with acetic acid $(60 \mathrm{~g} / \mathrm{l})$ overnight and stored in acetic acid $(60 \mathrm{~g} / \mathrm{l})$.

\section{Gel analysis}

Excess acetic acid was removed from the destained gels, which were placed on the flat-bed scanner (UMAX Vistascan S6, UMAX Data Systems Inc, Taipei, Taiwan) between two sheets of overhead transparency film and covered with a sheet of paper. The gels were scanned in reflective mode using Adobe Photoshop 3.0 software (Adobe Systems Incorporated, Mountain View, CA, USA) at 150300 dots per inch on a Macintosh computer (Apple Computer Inc., Cupertino, CA, USA). The image was then imported into the NIH Image software program, a public domain image processing program for the Macintosh (available to Internet users via anonymous FTP from zippy.nimh.nih.gov[128.231.98.32]). Density traces of each lane were obtained using gel plotting macros and the relative migration distances ( $\mathrm{R}_{\mathrm{f}}$-values) of the primary peaks calculated. Peak LDL particle diameters were determined from calibration curves of the $\log$ of molecular diameter of standards against $R_{f}$ using 2 nd polynomial equations. Standards were run on every gel and included apoferritin and thyroglobulin (diameters 12.2 and $17 \mathrm{~nm}$ respectively; Pharmacia, Uppsala, Sweden) and polystyrene latex beads ( $28 \mathrm{~nm}$, Duke Scientific Corp, CA, USA).

\section{Statistics}

Data are expressed as means \pm S.D. Methods were compared using Student's paired two-tailed $t$-test. Correlations were sought using the Pearson product moment correlation coefficient. A P value $<0.05$ was considered significant. All statistics analyses were conducted using Sigmastat software (Jandel Scientific, San Rafael, CA, USA).

\section{Results}

Examples of the density traces obtained from the gel scans are shown in figure 1. Isolated LDL (top four graphs) is usually seen as a single main peak, frequently with minor peaks and shoulders as described previously (8). When plasma is electrophoresed (lower four graphs of the same subjects), a peak corresponding to LDL is visible separated from smaller proteins which migrate further into the gel. The LDL peak may be linked to minor peaks closer to the origin: the latter include $\mathrm{Lp}(\mathrm{a})$, intermediate density lipoproteins (IDL), and very low density lipoproteins (VLDL) (9). These peaks are usually present in much lower concentrations than LDL, and rarely obscure the quantitatively more important LDL peak.

There was a good correlation between peak LDL particle diameters obtained using isolated LDL and whole plasma ( $\mathrm{r}=0.87, \mathrm{P}<0.001 ; \mathrm{n}=22$, fig. 2). However, the group means $(24.7 \pm 0.6$ and $24.8 \pm 0.5 \mathrm{~nm}$ respectively) were statistically different on the paired $t$-test $(\mathrm{P}<0.05)$.

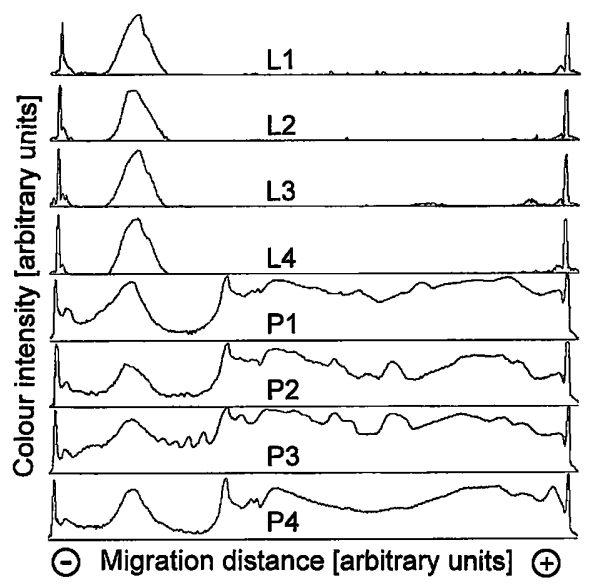

Fig. 1 Densitometric tracings of isolated LDL (L1-4) from four subjects and corresponding scans of unfractionated plasma samples from the same subjects (scans $\mathrm{P} 1-4$ respectively). The sharp peaks at the left and right of the tracings represent the top (origin) and bottom edges of the gel.

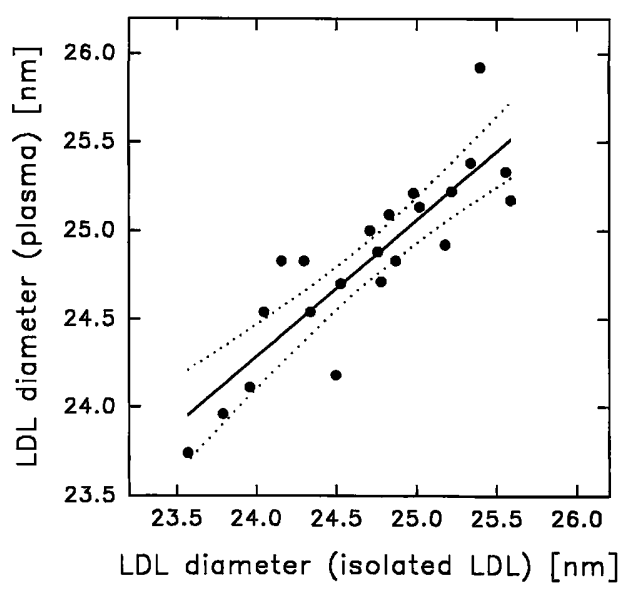

Fig. 2 Correlation between LDL diameters obtained by electrophoresing isolated LDL or plasma $(r=0.87 ; \mathrm{P}<0.001)$. The $95 \%$ confidence limits are shown by the dotted lines. The linear regression equation is $\mathrm{y}=5.7+0.77 \mathrm{x}$. 


\section{Discussion}

The isolation of LDL for analysis requires an ultracentrifuge, and conventionally involves prolonged centrifugation times (24-48 h). The use of plasma for LDL sizing avoids possible structural changes to the LDL during the purification procedure; however constituents of complex protein mixtures may interfere with the separation of individual proteins, especially if present in excessive concentrations. In the present case however, the principle lipoprotein of interest (LDL) is the primary large molecular mass particle present. As the scans show, proteins other than the lipoproteins migrate ahead of the LDL particle and consequently do not interfere with the migration of LDL as it reaches its size exclusion limit in the gel. Indeed, the electrophoresis of plasma has been widely used in the sizing of LDL particles (e. g. $(11,12))$, but the bands have been visualised using lipid stains. These stains e.g. Oil Red $O$ and Sudan Black are less convenient to use than protein stains, in that staining times are prolonged.

Lipoproteins with diameters larger than LDL, namely $\mathrm{Lp}(\mathrm{a})$, IDL and VLDL are found between the origin and the LDL peak $(11,13)$ but these proteins are generally present in much lower concentrations (13). Difficulties could arise if $\mathrm{Lp}$ (a) concentrations were greatly raised (as occurs in some patients as a genetic trait). However, $\mathrm{Lp}$ (a) bands are localized to positions above and separate from the LDL bands, and LDL is clearly the dominant band on electrophoresis (13).

The early study of Krauss \& Burke 1982 (8) suggested that there were comparable separations of LDL peaks

\section{References}

1. Austin MA, Breslow JL, Hennekens $\mathrm{CH}$, Buring JE, Willett WC, Krauss RM. Low-density lipoprotein subclass patterns and risk of myocardial infarction. J Am Med Assoc 1988; 26:1917-21.

2. Austin MA, King MC, Vranizan KM, Krauss RM. Atherogenic lipoprotein phenotype: a proposed genetic marker for coronary heart disease risk. Circulation 1990; 82:495-506.

3. Coresh J, Kwiterovich PO, Smith HH, Bachorik PS. Association of plasma triglyceride concentration and LDL particle diameter, density and chemical composition with premature coronary artery disease in men and women. J Lipid Res 1993; 34:1687-97.

4. Griffin BA, Freeman DJ, Tait GW, Thomson J, Caslake MJ, Packard CJ, et al. Role of plasma triglyceride in the regulation of low density lipoprotein (LDL) subfractions: relative contribution of small, dense LDL to coronary heart disease risk. Atherosclerosis $1994 ; 106: 241-53$.

5. Rajman I, Maxwell S, Cramb R, Kendall M. Particle size: the key to the atherogenic lipoprotein? Q J Med 1994; 87:709-20.

6. O'Neal D, Lee P, Murphy B, Best J. Low-density lipoprotein particle size distribution in end-stage renal disease treated with hemodialysis or peritoneal dialysis. Am J Kidney Dis 1996; 27:84-91.

7. Shen MS, Krauss RM, Lindgren FT, Forte TM. Heterogeneity of serum low density lipoproteins in normal human subjects. J Lipid Res 1981; 22:236-44.

8. Krauss RM, Burke DJ. Identification of multiple subclasses of plasma low density lipoproteins in normal humans. J Lipid Res $1982 ; 23: 97-104$. when plasma (visualised by lipid staining) or isolated LDL was used (8). The present study found a statistical difference in peak LDL particle diameter, with LDL sizes derived from plasma samples being larger than those derived from isolated LDL (means 24.8 versus $24.7 \mathrm{~nm}$ ). Whether matrix effects (protein interactions) were responsible for this difference is unclear. It has been suggested that changes in LDL characteristics during the isolation procedure may result in differences in particle sizes, but this was considered unlikely (8). The difference between the means of the two methods reported here is numerically small $(0.1 \mathrm{~nm})$ and may not be clinically important. Overall, the two methods are in good agreement $(\mathrm{r}=0.87, \mathrm{P}<0.001)$, and the simplified procedure readily lends itself to studies involving large numbers of samples.

\section{Conclusions}

This study shows that plasma stained with Coomassie Blue can be used for LDL particle size determinations by non-denaturing polyacrylamide gradient gel electrophoresis. Flat bed scanners and the NIH Image program provide a convenient means of scanning and analysing the gels. Plasma LDL particle diameters so obtained correlate well with measurements obtained on LDL isolated by ultracentrifugation.

\section{Acknowledgements}

We wish to sincerely thank Sr Colleen Morgan for subject liaison and organisation of sample collections.

9. Nichols AV, Krauss RM, Musliner TA. Nondenaturing polyacrylamide gradient gel electrophoresis. Methods in Enymology $1986 ; 128: 417-31$.

10. Sattler W, Bone P, Stocker R. Isolation of human VLDL, LDL, HDL and two subclasses in the TL-100 tabletop centrifuge using the TLA-100.4 rotor. Beckman Technical Information, 1992.

11. McNamara JR, Campos H, Ordovas JM, Peterson J, Wilson PWF, Schaefer EJ. Effect of gender, age, and lipid status on low density lipoprotein subfraction distribution. Results from the Framingham offspring study. Arteriosclerosis 1987; 7:483-90.

12. Princen HMG, van Duyvenvoorde W, Buytenhek R, van der Laarse A, von Poppel G, Leuven JAG, et al. Supplementation with low doses of vitamin E protects LDL from lipid peroxidation in men and women. Arterioscler Thromb Vasc Biol 1995; $15: 325-33$

13. McNamara JR, Campos H, Adolphson JL, Ordovas JM, Wilson WF, Albers JJ, et al. Screening for lipoprotein[a] elevations in plasma and assessment of size heterogeneity using gradient gel electrophoresis. J Lipid Res 1989; 30:747-55.

Received August 12/October 8, 1996

Corresponding author: Dr J. Westhuyzen, Conjoint Internal Medicine Laboratory, Royal Brisbane Hospital, Herston, Brisbane 4029, Australia 

要

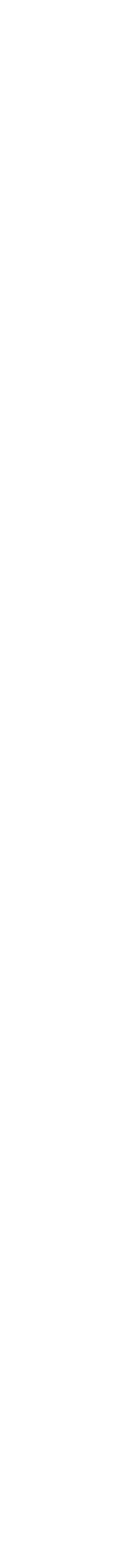

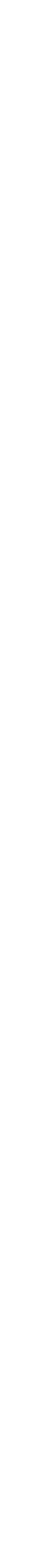

